CREAT. MATH. INFORM.

Volume 29 (2020), No. 2,

Pages 161 - 164

\title{
Some regularity properties of the functions obtained from some algebraic properties
}

\author{
DAN ŞTEFAN MARINESCU and MiHAi MONEA
}

ABSTRACT. The main result of this note is stated as follows. Let $a, b \in \mathbb{R}, a<b$, and a function $f:(a, b) \rightarrow \mathbb{R}$. We consider $u, v:(a, b) \rightarrow \mathbb{R}$ such that $u(x)>0$ and $v(x)<0$, for any $x \in(a, b)$, and define $g, h:(a, b) \rightarrow \mathbb{R}$ by $g(x)=u(x) f(x)$ and $h(x)=v(x) f(x)$, for any $x \in(a, b)$. The main result we establish is stated as follows:

Theorem. Let $n$ a positive integer, $n \geq 3$. If $u, v$ are $(n-1)$-times differentiable on $(a, b)$ and $g, h$ are $n$-convex functions then $f$ is $(n-1)$-times differentiable on $(a, b)$.

\section{INTRODUCTION}

The following contest problem, stated here as a Proposition 1.1, has been proposed to the 2018's district round of the Romanian National Mathematical Olympiad [7].

Proposition 1.1. Let $a, b \in \mathbb{R}, a<b$, and the function $f:(a, b) \rightarrow \mathbb{R}$, such that the functions $g, h:(a, b) \rightarrow \mathbb{R}$ defined by $g(x)=(x-a) f(x)$ and $h(x)=(x-b) f(x)$, for any $x \in(a, b)$, are nondecreasing. Then $f$ is continuous on $(a, b)$.

We remark that the reverse implication of Proposition 1.1 fails. For example, the function $f:(-1,1) \rightarrow \mathbb{R}, f(x)=|x|$ is continuous but the functions $g, h:(-1,1) \rightarrow \mathbb{R}$, defined by $g(x)=(x+1) f(x)$ and $h(x)=(x-1) f(x)$, for any $x \in(-1,1)$, are not monotone.

A similar result has been published later by the authors of this note, see [4].

Proposition 1.2. Let $a, b \in \mathbb{R}, a<b$, and the function $f:(a, b) \rightarrow \mathbb{R}$ such that the functions $g, h:(a, b) \rightarrow \mathbb{R}$ defined by $g(x)=(x-a) f(x)$ and $h(x)=(x-b) f(x)$, for any $x \in(a, b)$, are convex. Then $f$ is differentiable on $(a, b)$.

Starting from these interesting results, the aim of this note is to present some similar but more general results.

\section{ABOUT THE $n$-CONVEX FUNCTIONS}

The concept of $n$-convex function, where $n$ is a positive integer, is due to Hopf [1] and Popoviciu [6]. We remind that a function $f$, defined on the real interval $I$, is called $n$-convex if

$$
\begin{gathered}
\frac{f\left(x_{0}\right)}{\left(x_{0}-x_{1}\right)\left(x_{0}-x_{2}\right)\left(x_{0}-x_{n}\right)}+\frac{f\left(x_{1}\right)}{\left(x_{1}-x_{0}\right)\left(x_{1}-x_{2}\right)\left(x_{1}-x_{n}\right)}+\ldots \\
+\frac{f\left(x_{n}\right)}{\left(x_{n}-x_{0}\right)\left(x_{n}-x_{1}\right)\left(x_{n}-x_{n-1}\right)} \geq 0
\end{gathered}
$$

Received: 12.07.2019. In revised form: 18.02.2020. Accepted: 25.02.2020 2010 Mathematics Subject Classification. 26B25.

Key words and phrases. continuous functions, differentiable functions, convex functions, $n$-convex functions.

Corresponding author: Mihai Monea; mihaimonea@yahoo.com 
for any distinct $x_{0}, x_{1}, \ldots, x_{n}$ from the interval $I$.

It can be easily seen that a 1-convex function is a nondecreasing function while a 2convex function is convex in the usual sense.

Some properties of $n$-convex functions are similar to that of classical convex functions. Let $n$ a positive integer, $n \geq 3$ and $I$ an open interval. If $f: I \rightarrow \mathbb{R}$ is a $n$-convex function then $f$ is $(n-2)$-times differentiable on $I$ and the derivative $f^{(n-2)}$ is convex. Then, for any $x \in I$, there exist the finite one-sided $(n-1)$-th derivatives $f_{-}^{(n-1)}(x), f_{+}^{(n-1)}(x)$ and $f_{-}^{(n-1)}(x) \leq f_{+}^{(n-1)}(x)$.

More on this kind of results - which are not trivial - can be found in [2] and [3].

Now, we are in position to state a result that generalize the results from the previous section. First, let $n$ be a positive integer, $n \geq 3$ and $a, b \in \mathbb{R}, a<b$.

For any function $f:(a, b) \rightarrow \mathbb{R}$, we consider $g, h:(a, b) \rightarrow \mathbb{R}$ defined by $g(x)=$ $(x-a) f(x)$ and $h(x)=(x-b) f(x)$, for any $x \in(a, b)$.

Proposition 2.1. If $g$, $h$ are $n$-convex functions, then $f$ is $(n-1)$-times differentiable on $(a, b)$.

In the next section we will present the proof of this proposition as a consequence of a more general result.

\section{MAIN RESULTS}

Let $a, b \in \mathbb{R}, a<b$, and $f:(a . b) \rightarrow \mathbb{R}$ a function. We consider $u, v:(a, b) \rightarrow \mathbb{R}$ such that $u(x)>0$ and $v(x)<0$, for all $x \in(a, b)$ and define the functions $g, h:(a, b) \rightarrow \mathbb{R}$ by $g(x)=u(x) f(x)$ and $h(x)=v(x) f(x)$, for any $x \in(a, b)$.

Proposition 3.1. If $u, v$ are continuous on $(a, b)$ and $g, h$ are nondecreasing on $(a, b)$, then $f$ is continuous on $(a, b)$.

Proof. Let $c \in(a, b)$. We will prove that $\lim _{x \nearrow_{c}} f(x)=\lim _{x \searrow_{c}} f(x)=f(c)$. First, we observe that $\lim _{x \nearrow_{c}} f(x)$ and $\lim _{x \searrow c} f(x)$ exist due to the assumptions in the hyphotesis.

Now, for any $x \in(a, c)$, we have $g(x) \leq g(c)$, also $u(x) f(x) \leq u(c) f(c)$. We obtain $f(x) \leq \frac{u(c)}{u(x)} f(c)$. Hence $\lim _{x \nearrow c} u(x)=u(c) \neq 0$, then $\lim _{x \nearrow_{c}} f(x) \leq f(c)$. On the other side, we have $h(x) \leq h(c)$, also $v(x) f(x) \leq v(c) f(c)$. We obtain $f(x) \geq \frac{v(c)}{v(x)} f(c)$. Hence, $\lim _{x \nearrow_{c}} v(x)=v(c) \neq 0$, then $\lim _{x \nearrow_{c}} f(x) \geq f(c)$. As a consequence, we find $\lim _{x \nearrow_{c}} f(x)=f(c)$.

In the same way, by starting from $x \in(c, b)$, we obtain $\lim _{x \searrow c} f(x)=f(c)$.

The proof of the following proposition will use some properties of the convex functions, that can be found, for example, in [5]. We recall that if $f:(a, b) \rightarrow \mathbb{R}$ is a convex function, then $f$ is continuous on $(a, b)$ and there exist $f_{-}^{\prime}(x)$ and $f_{+}^{\prime}(x)$, for any $x \in(a, b)$. Moreover, they are finite and $f_{-}^{\prime}(x) \leq f_{+}^{\prime}(x)$, for any $x \in(a, b)$.

Proposition 3.2. If $u, v$ are differentiale on $(a, b)$ and $g$, $h$ are convex on $(a, b)$ then $f$ is differentiable on $(a, b)$.

Proof. Let $c \in(a, b)$. We will prove that $f_{-}^{\prime}(c)=f_{+}^{\prime}(c) \in \mathbb{R}$. From $g(x)=u(x) f(x)$, we obtain $f(x)=\frac{g(x)}{u(x)}$. Then there exist $f_{-}^{\prime}(x)$ and $f_{+}^{\prime}(x)$. Moreover, for any $x \in(a, b)$, we have

$$
f_{-}^{\prime}(x)=\frac{g_{-}^{\prime}(x) u(x)-g(x) u^{\prime}(x)}{u^{2}(x)}=\frac{g_{-}^{\prime}(x)}{u(x)}-\frac{g(x) u^{\prime}(x)}{u^{2}(x)}
$$


and

$$
f_{+}^{\prime}(x)=\frac{g_{+}^{\prime}(x)}{u(x)}-\frac{g(x) u^{\prime}(x)}{u^{2}(x)} .
$$

Hence $g_{-}^{\prime}(x) \leq g_{+}^{\prime}(x)$ and $u(x) \geq 0$, we obtain $f_{-}^{\prime}(x) \leq f_{+}^{\prime}(x)$.

In the same way, the equality $h(x)=v(x) f(x)$ lead us to

$$
f_{-}^{\prime}(x)=\frac{h_{-}^{\prime}(x)}{v(x)}-\frac{h(x) v^{\prime}(x)}{v^{2}(x)}
$$

and

$$
f_{+}^{\prime}(x)=\frac{h_{+}^{\prime}(x)}{v(x)}-\frac{h(x) v^{\prime}(x)}{v^{2}(x)},
$$

for any $x \in(a, b)$. Hence, $h_{-}^{\prime}(x) \leq h_{+}^{\prime}(x)$ and $v(x) \leq 0$, we obtain $f_{-}^{\prime}(x) \geq f_{+}^{\prime}(x)$.

We conclude that $f_{-}^{\prime}(x)=f_{+}^{\prime}(x)$.

The main result of this paper is given by the following theorem.

Theorem 3.3. Let $n$ be a positive integer, $n \geq 3$. If $u, v$ are $(n-1)$-times differentiable on $(a, b)$ and $g, h$ are $n$-convex function, then $f$ is $(n-1)$-times differentiable on $(a, b)$.

Proof. We will prove that $f_{+}^{(n-1)}(x)=f_{-}^{(n-1)}(x)$, for any $x \in(a, b)$.

From $f(x)=\frac{g(x)}{u(x)}$, we obtain that $f$ is $(n-1)$-times differentiable on $(a, b)$. Using Leibniz's formula for the derivatives of a product, we obtain

$$
\begin{aligned}
g^{(n-2)}(x) & =(u(x) f(x))^{(n-2)} \\
& =\sum_{k=0}^{n-3}\left(\begin{array}{c}
n-2 \\
k
\end{array}\right) u^{(n-2-k)}(x) f^{(k)}(x)+u(x) f^{(n-2)}(x),
\end{aligned}
$$

for any $x \in(a, b)$. Hence, $u^{(n-2)}$ is differentiable and $g^{(n-2)}$ is convex, then $f^{(n-2)}$ has one-sided derivatives, for any $x \in(a, b)$. Moreover, for any $x \in(a, b)$, we have

$$
\begin{aligned}
g_{-}^{(n-1)}(x)= & \sum_{k=0}^{n-3}\left(\begin{array}{c}
n-2 \\
k
\end{array}\right)\left(u^{(n-1-k)}(x) f^{(k)}(x)+u^{(n-2-k)}(x) f^{(k+1)}\right) \\
& +u^{\prime}(x) f^{(n-2)}(x)+u(x) f_{-}^{(n-1)}(x) .
\end{aligned}
$$

In the same way, we obtain

$$
\begin{aligned}
g_{+}^{(n-1)}(x)= & \sum_{k=0}^{n-3}\left(\begin{array}{c}
n-2 \\
k
\end{array}\right)\left(u^{(n-1-k)}(x) f^{(k)}(x)+u^{(n-2-k)}(x) f^{(k+1)}\right) \\
& +u^{\prime}(x) f^{(n-2)}(x)+u(x) f_{+}^{(n-1)}(x) .
\end{aligned}
$$

Then

$$
g_{+}^{(n-1)}(x)-g_{-}^{(n-1)}(x)=u(x)\left(f_{+}^{(n-1)}(x)-f_{-}^{(n-1)}(x)\right) .
$$

Hence, $g^{(n-2)}$ is convex, then $g_{+}^{(n-1)}(x) \geq g_{-}^{(n-1)}(x)$. We find

$$
f_{+}^{(n-1)}(x) \geq f_{-}^{(n-1)}(x),
$$

for any $x \in(a, b)$.

Starting from equality $h(x)=v(x) f(x)$, we obtain

$$
h_{+}^{(n-1)}(x)-h_{-}^{(n-1)}(x)=v(x)\left(f_{+}^{(n-1)}(x)-f_{-}^{(n-1)}(x)\right) .
$$


Hence $h_{+}^{(n-1)}(x) \geq h_{-}^{(n-1)}(x)$ and since $v(x)<0$, we find

$$
f_{+}^{(n-1)}(x) \leq f_{-}^{(n-1)}(x),
$$

for any $x \in(a, b)$. Then

$$
f_{+}^{(n-1)}(x)=f_{-}^{(n-1)}(x),
$$

for any $x \in(a, b)$ and the proof is complete.

Now, if we choose $u(x)=x-a$ and $v(x)=x-b$, we obtain the proof of the Proposition 2.1.

Finally, since a 1-convex function means that it is nondecreasing and a 2 -convex function means that it is convex, with the convention that 0-time differentiable means continuity, we can state the following unitary result.

Theorem 3.4. If the functions $u, v$ are $(n-1)$-times differentiable and $g, h$ are $n$-convex, then $f$ is $(n-1)$-times differentiable, for any positive integer $n$.

\section{REFERENCES}

[1] Hopf, E., Über die Zusammenhänge zwischen gewissen höheren Differenzenquotienten reeller Funktionen einer reellen Variablen und deren Differenzierbarkeitseigenschaften, PhD dissertation, Friedrich-Wilhelms-Universität, Berlin, 1926

[2] Karlin, S. and Studden, W. J., Tchebycheff Systems: With Applications in Analysis and Statistics, Pure Appl. Math., vol. XV, Interscience Publishers John Wiley \& Sons, New York, London, Sydney, 1966

[3] Kuczma, M., An Introduction to the Theory of Functional Equations and Inequalities, Birkhäuser Verlag AG, 2009

[4] Marinescu, D. Ş. and Monea, M., Problem 12096, Amer. Math. Monthly, 126 (2019), pag. 181

[5] Niculescu, C. P. and Persson, L.-E., Convex Functions and Their Applications, Springer, 2018

[6] Popoviciu, T., Sur quelques propriétés des fonctions d'une ou de deux variables réelles, Mathematica, 8 (1934), $1-85$

[7] ***, Romanian Mathematical Competitions, RMC 2018, Bucharest, 2018

Colegiul Naţional "IAncu de Hunedoara" Hunedoara, Romania

Email address: marinescuds@gmail.com

Colegiul Național "Decebal" Deva, Romania

Email address: mi haimonea@yahoo.com 